\title{
PHARMACIST-MANAGED THERAPEUTIC DRUG MONITORING SERVICE FOR ANTIEPILEPTIC DRUGS AND IMPROVED SEIZURE CONTROL
}

\author{
CHUN WAI CHANG ${ }^{*}$ AND SIVARAJ RAMAN \\ Hospital Keningau, Peti Surat 11, 89007 Keningau, Sabah, Malaysia
}

Published online: 23 October 2018

To cite this article: CHUN WAI CHANG \& SIVARAJ RAMAN (2018) Pharmacist-managed therapeutic drug monitoring service for antiepileptic drugs and improved seizure control, Malaysian Journal of Pharmaceutical Sciences, 16(1): 37-43, https://doi.org/10.21315/ mjps2018.16.1.3.

To link to this article: https://doi.org/10.21315/mjps2018.16.1.3

Although therapeutic drug monitoring (TDM) has been used in practice, conflicting data on its usefulness in the management of epilepsy have been reported. These results range from identifying no significant differences in patients' clinical outcomes to determining TDM to be a cost-effective service. Thus, this study was conducted to evaluate the effectiveness of our pharmacist-managed TDM service in helping patients with epilepsy (PWE) to achieve seizure control. This was a retrospective observational study conducted in the TDM Unit of Hospital Keningau, Sabah. Pharmacist-prepared reports issued for 30 subjects with uncontrolled seizures in 2014 were analysed to determine the effectiveness of their recommendations. Effectiveness was measured based on the number of patients who achieved $\geq 50 \%$ reduction in seizure frequency and the number of patients with a threemonth seizure-free period. Overall, $80 \%$ of the pharmacists' TDM recommendations were accepted by prescribers. Based on the data collected, 17 (56.67\%) subjects had their seizure frequency decreased at least by half, while 11 (36.67\%) subjects achieved total remission. However, there was no significant association between acceptance of recommendations and seizure control; although acceptance of pharmacist recommendations was associated with 1.4 times greater odds of achieving seizure control among PWE, this difference was not statistically significant. In conclusion, a pharmacist-managed TDM service was associated with an improvement in seizure control of more than 50\% among PWE with unsatisfactory seizure control.

Keywords: Therapeutic drug monitoring (TDM), Pharmacist, Effectiveness

\section{INTRODUCTION}

Therapeutic drug monitoring (TDM) is defined as the measurement of the concentration of a prescribed xenobiotic or endogenous compound made in the laboratory that, with appropriate interpretation, may directly influence prescribing procedures (Watson et al. 1997). TDM has been employed in the management of epilepsy since its introduction more than 40 years ago (Taur et al. 2013).

"Corresponding author: leckaschang@gmail.com

(C) Penerbit Universiti Sains Malaysia, 2018. This work is licensed under the terms of the Creative Commons Attribution (CC BY) (http://creativecommons.org/licenses/by/4.0/). 
In Malaysia, TDM services have been available since 1984 (Rahman, Abdelrahim and Ibrahim 2013). The first facility to offer this service was Hospital Universiti Sains Malaysia. In year 2006, there were 78 government hospitals in Malaysia offering TDM services (Rahman, Abdelrahim and Ibrahim 2013). Official records indicated that there were a total of 61,907 cases receiving TDM in Malaysia in 2005 alone (Rahman, Abdelrahim and Ibrahim 2013). In hospitals with TDM, this service is handled by pharmacists.

Although TDM has been used in practice for decades, conflicting data on its usefulness in the management of epilepsy have been reported. A multicentre randomised controlled trial conducted by Januzzi et al. (2000) found no significant differences in clinical outcomes in patients with newly diagnosed epilepsy. However, two pharmacoeconomic studies in India (Rane et al. 2001) and Malaysia (Salih et al. 2013) demonstrated TDM to be cost effective in patients with adult onset epilepsy and in a specific paediatric population, respectively. The authors of a review identifying no clear evidence that supported the use of TDM in patients with newly diagnosed epilepsy nonetheless suggested that TDM may be useful during polytherapy, in special situations or in selected patients (Tomson, Dahl and Kimland 2007).

In our hospital, TDM services are provided by the pharmacy department. Prescribers identify patients with epilepsy (PWE) who require the service and refer them to hospital pharmacists. The frequency of seizure attacks, compliance and experience of side effects are elicited from patients. If the patient is admitted to the ward, the attending pharmacist also monitors the progress of the patient in the ward. Monitoring results are interpreted by pharmacists, and a report with recommendations for each patient is issued to the ordering prescriber. The prescriber then decides to accept or reject these recommendations based on their clinical judgement. The antiepileptic drugs (AEDs) that are monitored in our hospital include carbamazepine, phenobarbitone, phenytoin and valproic acid.

As the effects of TDM on clinical outcomes in PWE have not been fully elucidated, we conducted this study to evaluate the effectiveness of our pharmacist-managed TDM service in helping PWE to achieve seizure control.

\section{METHODS}

\section{Study Design}

This was a retrospective observational study conducted in the TDM Unit at Hospital Keningau, Sabah, Malaysia.

PWE were referred for TDM service when the prescriber deemed it was necessary. Monitoring results were interpreted by pharmacists, and a report with recommendations was provided to the ordering prescriber. The prescriber then accepted or rejected these recommendations based on their clinical judgement. The AEDs that were monitored included carbamazepine (reference range: 4-12 mg/L), phenobarbitone (15-40 mg/L), phenytoin (10-20 mg/L) and valproic acid (50-100 mg/L).

Data were obtained from 2014 TDM reports. Types of data that were collected included age, gender, type of AEDs, dates of sampling, seizure frequency, measured plasma levels of antiepileptic drugs and the status of recommendations (accepted or rejected by the ordering prescriber). 


\section{Subjects}

We included a total of 30 subjects in this study ( 37 subjects met the inclusion criteria, but seven subjects were excluded due to incomplete data).

The inclusion criteria were as follows: (1) PWE who had at least two TDM reports, (2) PWE with at least a three-month interval between the two reports and (3) PWE who, at the first sampling point, had uncontrolled seizures (having at least one seizure during the months preceding the sampling point).

The exclusion criterion was insufficient data on the TDM request form (including age, gender and seizure frequency).

\section{Effectiveness Measures}

The effectiveness measures employed by Salih et al. (2013), including the number of patients who achieved $\geq 50 \%$ reduction in seizure frequency and the number of patients with a three-month seizure-free period, were used in this study. Seizure control in patients was categorised as uncontrolled (defined as no change or an increase in seizure frequency), controlled (seizure frequency reduction of at least $50 \%$ ) and complete seizure remission according to the number of seizures self-reported by the patient. Seizure frequency was defined as the average number of seizures per month. If this definition could not be feasibly applied, seizure frequency was defined as the number of seizures experienced during the month preceding blood sampling. For statistical analysis, patient seizure control was categorised only as controlled or uncontrolled.

\section{Statistical Analysis}

The normality of all continuous variables was tested using the Shapiro-Wilk test. Further confirmation of normality was achieved via inspection of histograms.

We expressed normally distributed continuous sociodemographic variables as the means \pm SDs. Non-normally distributed continuous variables were reported as medians (IQRs). All nominal data were expressed as frequencies and percentages.

The association between the number of recommendations accepted and seizure control was analysed using Fisher's exact test, and phi coefficients were used to determine whether a result was statistically significant.

We also reported the odds ratio for controlled seizure status when a pharmacist's recommendation was accepted.

\section{Ethical Approval}

Ethical clearance was obtained from the Medical Research and Ethics Committee, Ministry of Health, Malaysia ([5]KKM/NIHSEC/P15-1050). 


\section{RESULTS}

\section{Demographic Data}

The demographic data of the subjects are presented in Table 1. All patients had been taking the specified AEDs for at least six months prior to study initiation. There were more male than female participants, and two-thirds of the subjects were on monotherapy.

Table 1: Demographic data $(n=30)$.

\begin{tabular}{lc}
\hline Age (year, median [IQR]) & $23(16.25,32.75)$ \\
Gender & frequency $(\%)$ \\
Male & $20(66.67 \%)$ \\
Female & $10(33.33 \%)$ \\
Number of antiepileptic drugs (frequency [\%]) & \\
1 & $20(66.67 \%)$ \\
2 & $8(26.67 \%)$ \\
3 & $2(6.66 \%)$ \\
Type of antiepileptic drug (frequency [\%]) & \\
Carbamazepine & $5(16.67 \%)$ \\
Lamotrigine & $3(10.00 \%)$ \\
Phenobarbitone & $1(3.33 \%)$ \\
Phenytoin & $7(23.33 \%)$ \\
Sodium valproate & $26(86.67 \%)$ \\
\hline
\end{tabular}

Notes: ${ }^{a}$ The total percentage is greater than $100 \%$, as some subjects were taking more than one antiepileptic.

\section{Outcome after TDM}

A total of $17(56.67 \%)$ subjects had their seizure frequency decreased by at least $50 \%$ after receiving TDM. Eleven (36.67\%) subjects achieved total remission after receiving TDM.

A total of 41 tests were performed during the two visits. There was an increase of $7.32 \%$ in the number of patients with drug concentrations within the predefined reference ranges after receiving TDM (Figure 1).

\section{Relationship between Accepted Recommendation and Seizure Control}

Of the 30 recommendations given, 24 were accepted, at an acceptance rate of $80 \%$. No statistically significant association was identified between the number of recommendations accepted by a prescriber and seizure control in the subjects ( $p=1.003$, Fisher's exact test). In the group of patients for which recommendations were accepted $(n=24)$, $58.33 \%$ achieved seizure control. In group for which recommendations were rejected $(n=6)$, this percentage was $50.00 \%$. Thus, the odds ratio of achieving seizure control was 1.4, 95\% Cl: 0.23-8.42. 


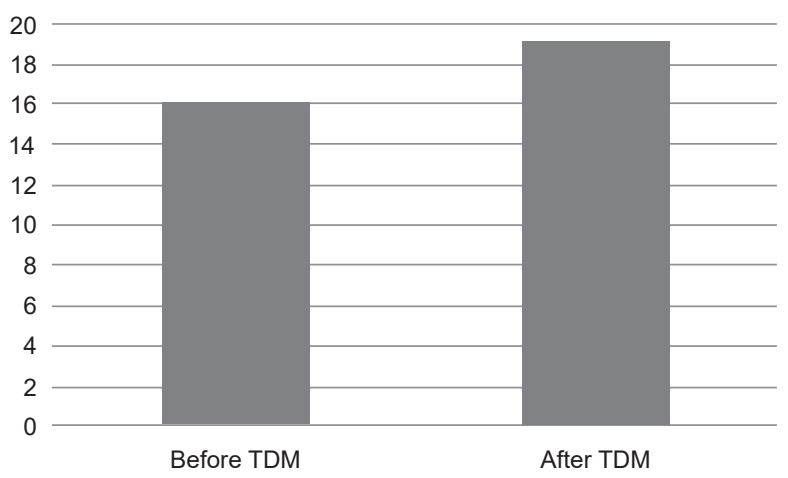

Fig. 1: Number of patients with drug concentrations within predefined reference ranges before and after receiving TDM.

\section{DISCUSSION}

Based on our results, more than half of the subjects achieved seizure control, and the majority of these subjects had a three-month seizure-free period after receiving the TDM service. Although the rate of accepting recommendations was high, we did not find any statistically significant association between the acceptance rate and seizure control. Patients for whom pharmacist recommendations were accepted were found to have a 1.4 times higher odds of achieving seizure control; however, this difference was not statistically significant.

We found that seizure control in subjects improved after receiving the TDM service, and most of the recommendations made were accepted by prescribers. This improvement in seizure control was consistent with the findings of studies conducted by Salih et al. (2013) and Rane et al. (2001), in which interpretations of TDM results were provided. However, no association between the acceptance of recommendations and seizure control was identified. Similarly, the odds ratio indicated a modest but not statistically significant increase in the odds of achieving seizure control when the pharmacist's recommendations were accepted.

Improvement in seizure control was observed both with and without the acceptance of pharmacist recommendations. We speculate that the factor most associated with this improvement was the counselling service provided by the pharmacists. During the encounters with PWE, the pharmacists might have provided motivation and reassurance, thus reinforcing compliance in both groups of subjects.

Based on our findings, recommendations provided by pharmacists may not be a critical factor in achieving better seizure control. Possible explanations for this observation include factors that may affect the quality of recommendations, such as the expertise of the pharmacist, seizure type and compliance of the PWE. Different pharmacists were involved in the TDM service; therefore, the quality of recommendations might have been inconsistent, as recommendation quality likely depended on the experience and knowledge of the pharmacist. Some subjects might have had seizure types that were difficult to control with AEDs alone. Thus, in these patients, pharmacist recommendations would likely have a limited effect on seizure control. Another explanation for the finding might be the noncompliance of the subjects. Even though noncompliance with the evaluated medications was identified during the service and the subjects had been counselled, seizure attacks associated with noncompliance remained unavoidable. 
In addition, the subjects were not proportionately distributed between the two groups; only six subjects were in the rejected recommendation group. This might have prevented the discernment of a significant difference in the outcome. Unfortunately, this disproportionality was unavoidable due to the high recommendation acceptance rate and prescribers' clinical judgement coinciding with the suggested recommendations.

The increase observed in the number of patients with drug concentrations within the predefined reference ranges after receiving the TDM service was consistent with the findings of the study conducted by Januzzi et al. (2000). Although there was an increase in the number of patients with drug concentrations within the predefined reference ranges, more than half of the plasma concentration measurements were still outside the reference range, but seizure control improved in more than $50 \%$ of subjects. This finding shows that these predefined reference ranges may not be good measures of clinical response. Due to individual variations, therapeutic benefits may be achieved at serum drug concentrations outside these ranges (Patsalos et al. 2008). The use of a therapeutic range that is defined by the range of drug concentrations that provide the best achievable response in a given patient population has been advocated (Patsalos et al. 2008). Thus, therapeutic ranges may differ between individuals (Patsalos et al. 2008).

In our study, the AED most frequently requested for TDM service was sodium valproate. Due to its wide therapeutic range and the variable association between its serum level and clinical response, it has been suggested that its dosage should be individualised on clinical grounds alone (Januzzi et al. 2000). TDM may still be valuable for phenytoin, phenobarbitone and carbamazepine for pharmacokinetic reasons (Januzzi et al. 2000; Patsalos et al. 2008; Touw et al. 2005). Nonetheless, TDM can be employed to determine the therapeutic range specific to each patient as a target for dosage adjustments and as a way to identify noncompliance in the event of future seizure attacks (Patsalos et al. 2008).

Based on the findings of our study, it can be observed that the presence of measured drug concentrations within the reference range does not predict seizure control. In our setting, which is similar to most hospitals in Malaysia, the time interval between appointments with doctors for PWE may be quite long. However, PWE have to collect their medications from the pharmacy monthly. Thus, we suggest that pharmacists monitor the condition of PWE during these visits to obtain an accurate picture of their clinical progress. By taking into account clinical conditions, a better interpretation of the measured drug concentrations can be achieved, and a more appropriate recommendation can be provided.

There are a few limitations of our study. First, the sample size was small. There might be recall bias, as seizure frequency was self-reported by the subjects. In addition, the type of seizure affecting the subjects was not recorded. Some seizure types are inherently more difficult to control, and this might have led to underestimation of the value of TDM.

\section{CONCLUSION}

Our study showed that a pharmacist-managed TDM service was associated with improved seizure control in more than $50 \%$ of PWE with unsatisfactory seizure control. Impactful recommendations depend on the competency of pharmacists and factors such as seizure type. Instead of a standardised reference range, an individualised therapeutic range may be more valuable for the management of PWE.

Malay J Pharm Sci, Vol. 16, No. 1 (2018): 37-43 


\section{ACKNOWLEDGEMENTS}

We would like to thank the Director of Health Malaysia for permission to publish this paper.

\section{REFERENCES}

JANUZZI, G., CIAN, P., FATTORE, C., GATTI, G., MONACO, F. \& PERUCCA, E. (2000) A multicenter randomized controlled trial on the clinical impact of therapeutic drug monitoring in patients with newly diagnosed epilepsy, Epilepsia, 41(2): 222-230. https://doi.org/10.1111/j.1528-1157.2000.tb00144.x.

PATSALOS, P. N., BERRY, D. J., BOURGEOIS, B. F. D., CLOYD, J. C., GLAUSER, T. A., JOHANNESSEN, S. I., et al. (2008) Antiepileptic drugs: Best practice guidelines for therapeutic drug monitoring; A position paper by the subcommission on therapeutic drug monitoring, ILAE Commission on Therapeutic Strategies, Epilepsia, 49(7): 1239-1276. https://doi.org/10.1111/j.1528-1167.2008.01561.x.

RAHMAN, A. F. A., ABDELRAHIM, H. E. A. \& IBRAHIM, M. I. M. (2013) A survey of therapeutic drug monitoring services in Malaysia, Saudi Pharmaceutical Journal, 21(1): 1924. https://doi.org/10.1016/j.jsps.2012.01.002. https://doi.org/10.1016/j.jsps.2012.01.002.

RANE, C. T., DALVI, S. S., GOGTAY, N. J., SHAH, P. U. \& KSHIRSAGAR, N. A. (2001) A pharmacoeconomic analysis of the impact of therapeutic drug monitoring in adult patients with generalized tonic-clonic epilepsy, British Journal of Clinical Pharmacology, 52(2): 193195. https://doi.org/10.1046/j.0306-5251.2001.01436.x.

SALIH, M. R. M., BAHARI, M. B., SHAFIE, A. A., HASSALI, M. A. A., AL-LELA, O. Q. B., ABD, A. Y., et al. (2013) Cost-effectiveness analysis for the use of serum antiepileptic drug level monitoring in children diagnosed with structural-metabolic epilepsy, Epilepsy Research, 104(1-2): 151-157. https://doi.org/10.1016/j.eplepsyres.2012.09.012.

TAUR, S. R., KULKARNI, N. B., GOGTAY, N. J. \& THATTE, U. M. (2013)An audit of therapeutic drug monitoring services of anticonvulsants at a tertiary care hospital in India, Therapeutic Drug Monitoring, 35(2): 183-187. https://doi.org/10.1097/FTD.0b013e3182803d16.

TOMSON, T., DAHL, M. L. \& KIMLAND, E. (2007) Therapeutic monitoring of antiepileptic drugs for epilepsy (review), Cochrane Database of Systematic Review, 2: CD002216. https://doi.org/10.1002/14651858.CD002216.pub2.

TOUW, D. J., NEEF, C., THOMSON, A. H. \& VINKS, A. A. (2005) Cost-effectiveness of therapeutic drug monitoring: A systematic review, Therapeutic Drug Monitoring, 27(1): 10 17. https://doi.org/10.1097/00007691-200502000-00004.

WATSON, I., POTTER, J., YATSCOFF, R., FRASER, A., HIMBERG, J. J. \& WENK, M. (1997) Editorial: Therapeutic drug monitoring, Therapeutic Drug Monitoring, 19(2): 125. https://doi.org/10.1097/00007691-199704000-00001. 